\title{
Effect of Chemical Composition and Heat Treatment on the Shape Memory Parameters in the TiNi-Me Alloys
}

\author{
V.I. Kolomytsev, A.V. Kozlov, R.Ya. Musienko and V.K. Soolshenko \\ Institute of Metal Physics, National Academy of Sciences of Ukraine, 36 Vernadskogo St., 252142 Kiev, \\ Ukraine
}

\begin{abstract}
The TiNi-Me shape memory alloy parameters (namely, phase transformation "strength yield", recoverable strain, reversion stress, material hardness) have been investigated as a function of the chemical composition, heat treatment regimes and deformation condition. These parameters are found to lke structurally sensitive ones both to the macroscopic and microscopic structure of the material. Their response to heat treatment regimes is usually non-homogeneous function of the aging temperature and time variatior. Effects of doping and secondary particle precipitation are of great importance. Some recommendations for the choice of the SMA chemical composition and final heat treatment regime can be proposed.
\end{abstract}

\section{INTRODUCTION}

The TiNi-base shape memory alloys (SMA's) remain to be the most attractive ones for application purposes due to good coincidence of strength, plasticity, corrosion ability, etc. Moreover, the required property parameters can be improved to a certain degree by appropriate combination of the production technology scheme, subsequent thermal treatment regimes and/or by third element doping [1-5]. Substantial disadvantage of these alloys is degradation of the martensite transformation parameters durings multiple thermal cycling under fixed load and/or stress cycling at fixed temperature.

This article presents some experimental data related to the TiNi-Me SMA's deformation parameters as a function of the chemical composition and preliminary heat treatment regime. Namely, the phase transformation "strength yield", recoverable strain, reversion stress developed during reverse transformation, transformation temperatures and their relation to the material chemical composition and structure are under consideration oriented on stabilization of the chosen shape memory parameters.

\section{MATERIAL AND EXPERIMENTAL TECHNIQUES}

The TiNi based SMA's were prepared by induction melting in inert gas atmosphere from iodide titanium (purity $99,8 \%$ ), electrolytic nickel $(99.99 \%$ ) and other raw materials of chemical purity. To achieve good homogeneity the ingots were three-times remelted.

If not other stated, prior to any measurements all specimen was normalized at $\sim 1270 \mathrm{~K}$ for $20 \mathrm{~min}$. after cold working and sample preparation procedures. Some of them were additionally treated at intermediate temperatures $\mathrm{T}=500-1000 \mathrm{~K}$ for various tempering periods $\mathrm{t}>10 \mathrm{~min}$.

The transformation temperatures were determined by the measurement of various physical property anomalies in the phase transformations temperature range, namely electrical resistance temperature variation in stress-free condition, bending versus temperature tests, unidirectional deformation measurements etc. Transformation temperatures in ternary TiNi-Me alloys are discussed elsewhere [5]. Three-points bending tests were carried out on samples size $3 \times 0.6 \times 35 \mathrm{~mm}$; deformation parameters were obtained in unidirectional tensile tests on samples size $4 \times(0.6-1.0) \mathrm{mm}$ and working length $20 \mathrm{~mm}$. Reversion stress was measured by strain-gauge attached to the elastic elements of the leads. 


\section{RESULTS AND DISCUSSION}

Let us characterize some parameters of the stress-strain curves obtained in tensile tests for the B2 $\angle$ B 19' and $\mathrm{B} 2<=>\mathrm{R} \Leftrightarrow \Rightarrow \mathrm{B} 19^{\prime}$ transformation sequences, similar to those presented in fig. 1 for Ti-50.1Ni and Ti$51 \mathrm{Ni}$ alloys. Usually several deformation stages are observed on the $\sigma-\varepsilon$ curves as a function of the test temperature variation around the martensite transformation temperature range. At a test temperature near $M_{d}$ the material deformation is mainly due to generation and motion of dislocations in the parent $\beta$ phase. Lowering of the test temperature to $A_{f}<T<M_{d}$ results pseudoelastic deformation caused by the formation of the stress-induced martensite. This deformation channel becomes predominant on lowering the test temperature to $M_{s}$ and below and its contribution to accumulated strain increases up to $\sim 4 \%$. So-called phase transformation "yield strength" $\sigma_{\text {ph., }}$ defined as it is shown in fig. 1, decreases almost linearly with lowering the test temperature slightly below $\mathrm{M}_{\mathrm{s}}$ (fig. 2).

It was found that the temperature slope of the B2->B19' martensite transformation "yield strength", $\mathrm{d} \sigma^{\mathrm{M}}{ }_{\mathrm{ph}} / \mathrm{dT}$, depends on the alloy chemical composition: it decreases with nickel content and on alloying by its chemical analogous $\mathrm{Cr}, \mathrm{Fe}, \mathrm{Co}$ (instead of $\mathrm{Ti}$ ). Phenomenological consideration of the Clausius-Clapeyron equation shows this effect can be attributed partly to the increase of the material density, perhaps to a decrease of transformation volume dilatation $\Delta \mathrm{V} / \mathrm{V}$. Increase of the material strength should be also taking into account (al least, microhardness measurement evidence it, see below).

Relatively large size of polycrystalline samples didn't allow to receive reliable numerical data of deformation behaviour at the $B 2 \Leftrightarrow R$ transition. Nevertheless, the tendency in the SME parameters was found to be similar to results presented by $[6,7]$ on wire and single crystal samples. Aging at intermediate temperatures $\left(500-1000 \mathrm{~K}\right.$ ) leads to separation of the $\mathrm{B} 2 \Leftrightarrow \Rightarrow \mathrm{R}$ and $\mathrm{R} \Leftrightarrow \Rightarrow \mathrm{B} 19^{\prime}$ transformations in binary and ternary TiNi-based SMA's [3]. Main feature of the stress-strain curves is the pseudoelastic plateau observed in samples after specific heat treatment, up to $3 \%$ length, with small deformation strengthening

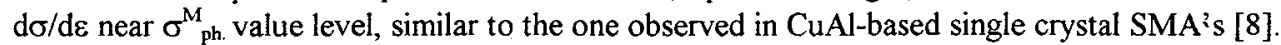

Value of the temperature slope $d \sigma^{\mathrm{R} h} / \mathrm{dT}$ for $\mathrm{B} 2->\mathrm{R}$ transition is higher than $\mathrm{d} \sigma^{\mathrm{M}}{ }_{\mathrm{ph}} / \mathrm{dT}$ fo: $\mathrm{B} 2=\mathrm{B} 19^{\prime}$ or $\mathrm{R} \Rightarrow \mathrm{B} 19^{\prime}$ transformations. This difference is an expected phenomenon since the transformation volume dilatation for the former transition is significantly lower than for others.

The elastic deformation of martensite, $\varepsilon_{\text {el. }}$, increases gradually with true strain. Recovery strai $i$, $\varepsilon_{\text {rec., }}$ associated with reverse martensite transformation, increases up to $4-5 \%$ with true strain up to $7 \%$;. Residual strain part, $\varepsilon_{\text {res, }}$, increases linearly with the degree of the specimen deformation up to $\sim 4 \%$ but for a higher deformation level there appears a nonlinear contribution. The definite part of the residual strain is caused by the martensite stabilization effect since its value decreases a little on heating to higher temperatures. Shift of the reverse transformation temperature interval to higher temperatures after deformation in martensite is usually observed in many SMA's. E.g., for Ti-50.1Ni alloy, the main part of the reverse transformation temperatures interval $A_{s}-A_{f}$ shifts from $300-350 \mathrm{~K}$ up to $390-450 \mathrm{~K}$ after $3 \%$ tension but presence of $5-7 \%$ stabilized martensite is observed up to $620 \mathrm{~K}$ by $\mathrm{X}$-ray photography.

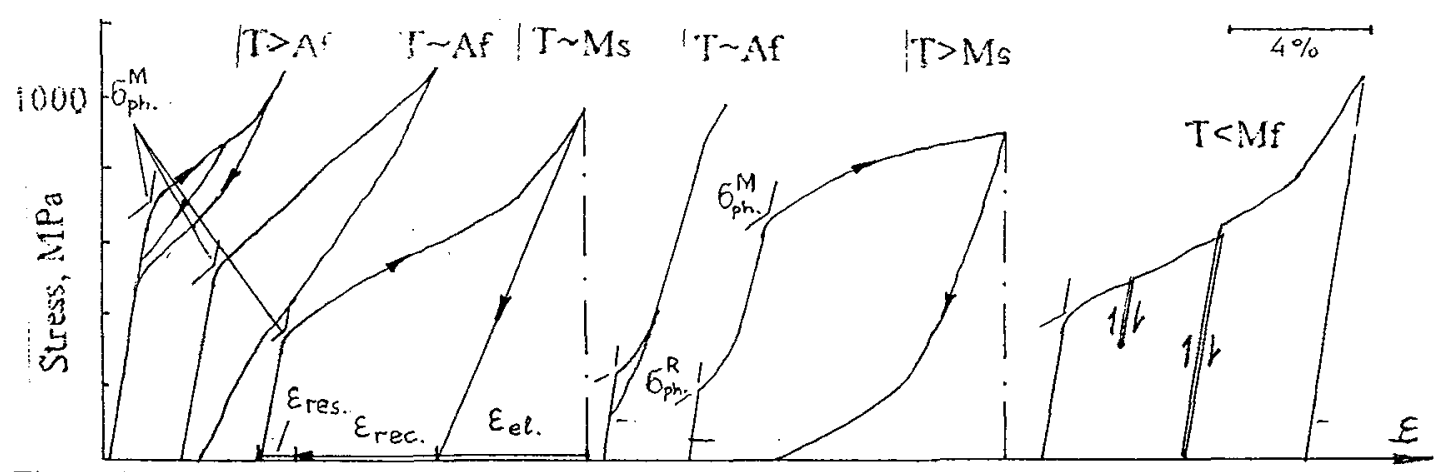

Figure 1: Typical the $\sigma-\varepsilon$ curves for $B 2<\Rightarrow B 19^{\prime}$ (left and righr, Ti-50.1Ni) and $B 2 \Leftrightarrow \Rightarrow R \Leftrightarrow B 19^{\prime}$ (niddle, Ti-51 N1) 


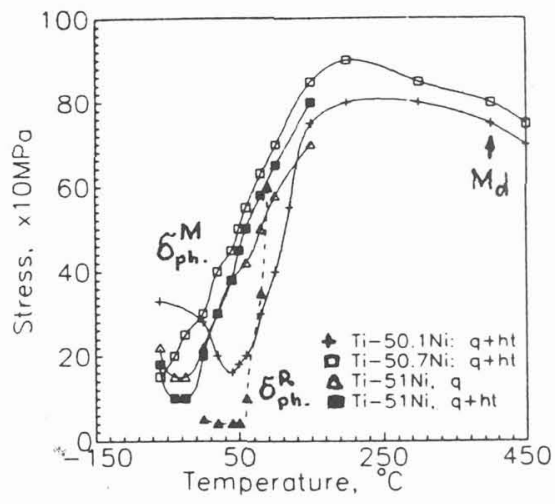

Figure 2: The phase transformation "strength yield" $\sigma_{\mathrm{ph}}$ versus test temperature dependence, $\mathrm{ht}: \mathrm{T}=720 \mathrm{~K}, \mathrm{t}=30 \mathrm{~min}$.

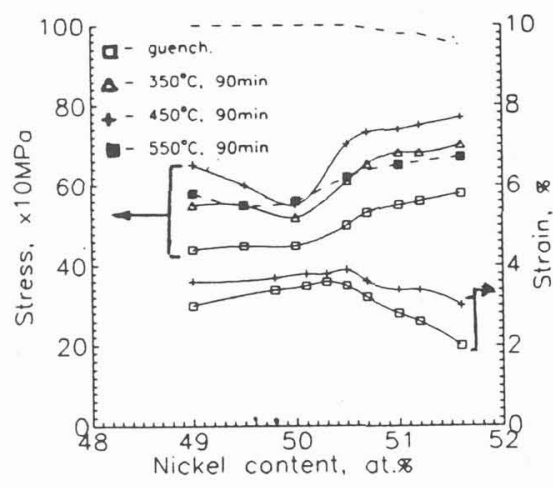

Figure 3: Reversion stress and fully recoverable strain (residual strain negligible) with alloy chemical composition and treatment regime binary TiNi SMA's.
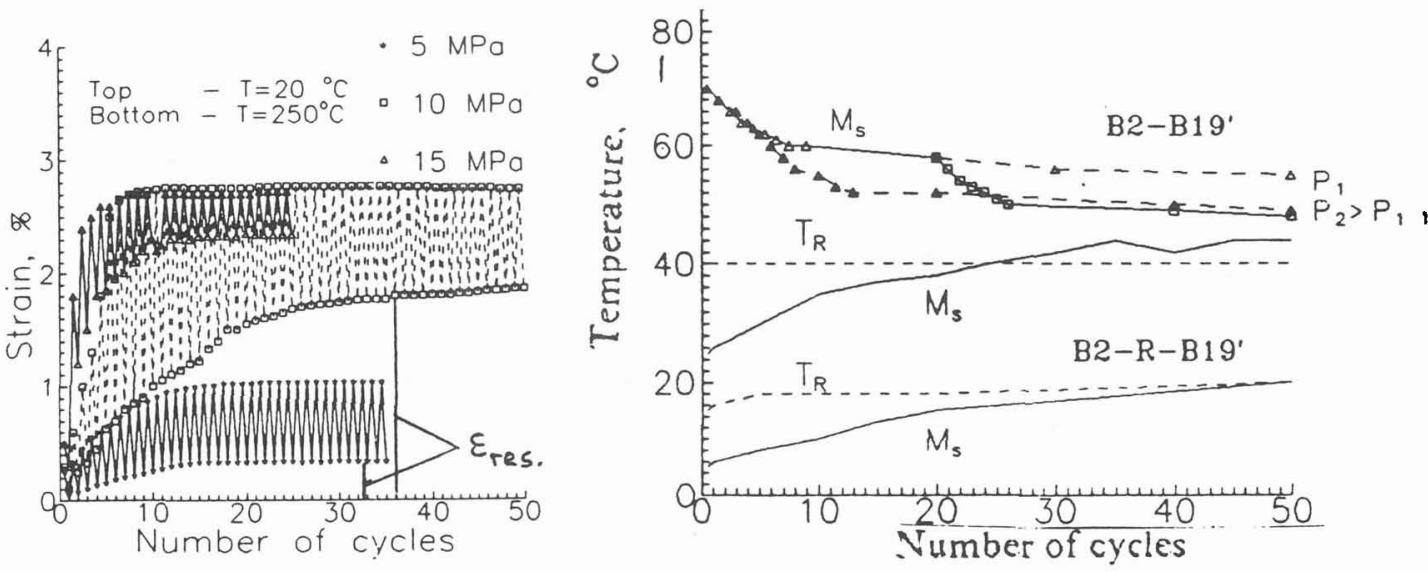

Figure 4: Deformation behaviour during full thermal Figure 5: Shiff of the transformation temperatures in cycling under fixed load in Ti-50.1Ni alloy.

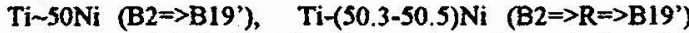

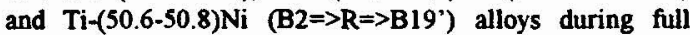
thermal cycling under various fixed load.
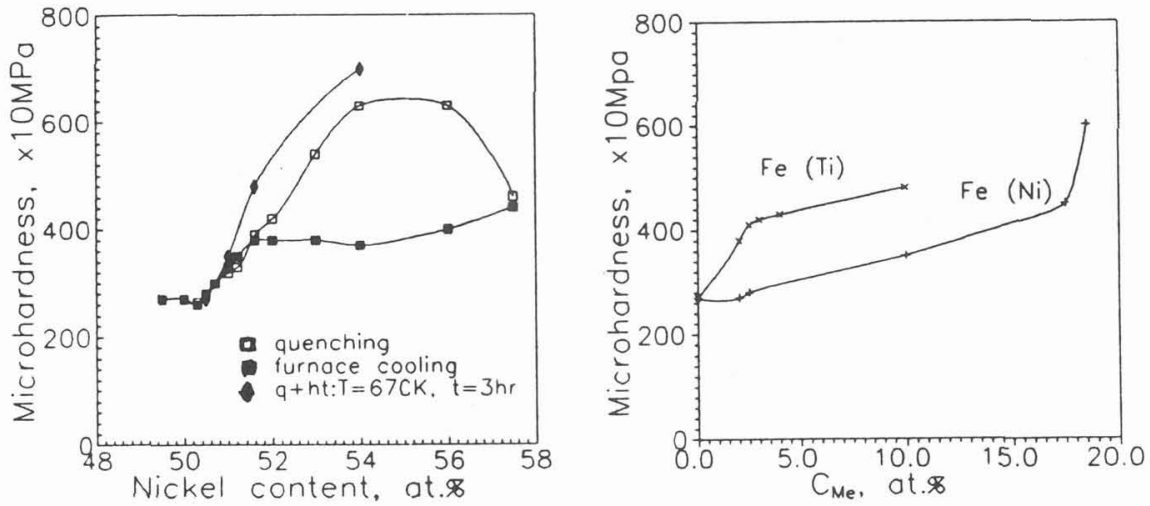

Figure 6: Microhardness value variation with alloy chemical composition in binary (a) and ternary (b) TiNi-based SMA's. 
Reversion stress developed by a specimen during reverse martensite transformation $\left(\sigma_{\mathrm{r}}\right)$ was measured in constrained condition after preliminary tension up to $7 \%$. Reversion stress was found to increase with degree of martensite preliminary deformation, reaches its highest values at $\varepsilon_{\text {tot. }}=2-3 \%$ and decreases $10-15 \%$ for $\varepsilon_{\mathrm{tot}}>3 \%$. Moreover, decrease of reversion stress and increase of residual strain take place on lowering the deformation test temperature below $\mathbf{M}_{\mathbf{f}}$.

Usually reversion stress does not reach the value of the "the phase transformation yield strength", $\sigma_{\mathrm{r}}<\sigma_{\mathrm{ph}}$. Some difference in values between $\sigma_{\mathrm{r}}$ and $\sigma_{\mathrm{ph}}$ is similar to Baushinger effect observed for strength yield on changing direction of deformation (e.g., compression versus tension).

Reasons for the reversion stress variation with deformation test temperature seem to be simple ones taking into consideration the increased stress level in material during deformation and subsequent its relaxation by generation of irrecoverable defects. In case of SMA's there appears additional deformation path by martensite formation, reorientation of martensite crystals and substructure in addition to customary relaxation mechanisms. So, it looks that for deformation test temperatures close to $M_{s}$ the generation of defects is minimal and stress relaxation takes place by the formation of martensite crystal ensemble, thus giving a higher value of a reversion stress closer to $\sigma_{\mathrm{ph} .}$. Nevertheless, generation of irrecoverable defects and accompanied residual strain occurs even on tension for optimal conditions (namely, when test temperature is slightly below $\mathbf{M}_{\mathbf{s}}$ or in monophase martensite state near $\mathbf{M}_{\mathrm{f}}$ ). Moreover, material fracture takes place just around the $M_{s}$ temperature for a lower stress applied $[9,10]$. Deformation of martensite for a given strain at $T<M_{\mathbf{f}}$ requires higher stress for a martesite reorientation process. Higher stress leads to the generation of defects in martensite thus lowering the reversion stress during subsequent reverse martensite transformation. Beside above mentioned reasons certain contributions to reversion stress may be caused by the change of reverse martensite transformation path with respect to the direct one.

It was found out in as-quenched Ti-Ni SMA's that the reversion stress increases almost linearly with nickel content in the $\beta$-phase homogeneity range (fig. 3). Isochronous aging leads to overall increase of the reversion stress, but its increment depends on alloy chemical composition and aging parameters (temperature and aging time) (fig. 3). This difference in material reversion stress response on agirig parameters is supposed to be a function of the predominate structure changes.

Deformation behaviour in bending under constant load condition in heating/cooling stages trougin the temperature intervals of the $\mathrm{B} 2 \Leftrightarrow \mathrm{R} \Leftrightarrow \Rightarrow \mathrm{B} 19^{\prime}$ martensite transformations also shows two step strain variation related to these transformations. Relative contribution of strain accumulated during $B 2->\mathrm{R}$ and $\mathrm{R}->\mathrm{B} 19^{\prime}$ to full recoverable strain depends on alloy chemical composition and preliminary heat treatment regimes $[4,11]$. Interesting results have been obtained concerning the degradation of the shape memor parameters during thermal cycling under fixed load. A certain residual strain $\varepsilon_{\text {res. }}$ remains usually in the material after full thermal cycles through the phase transformation region. Its value depends on the fixed load applied (fig. 4) (usually the total load does not produce a stress higher than $20 \%$ of yield strength), increases gradually with number of thermal cycles and stabilizes at a definite value for a given fixed load after up to 20 full thermal cycles.

Variation of the transformation temperatures $M_{s}$ and $T_{R}$ with the number of full thermal cycle; under fixed load has been studied (fig. 5). For B2->B19' transformation sequence the $M_{s}$ temperature usually decreases with number of thermal cycles and stabilizes after approximately 10-15 cycles. For the $B 2<=>R \Leftrightarrow B 19$ ' transformation sequence $M_{s}$ temperature increases gradually while $T_{R}$ temperature varies slowly with number of thermal cycles. A set of parallel dislocation lines were observed in TEMA bright-field images of samples after multiple thermal cycling. These results are in good agreement with observations described in [12].

To interpret the reversion stress and recoverable strain increase in samples treated at intermediate temperatures one should takes into account the complexity of the structural changes both of the parent and transformation product phases. Namely, possible changes in the $\beta$ phase grain size, type and degree of long range order, density of structural defects, secondary particle precipitates and their interaction with growing martensite plates, variation in transforming matrix chemical composition, changes in the martensite crystal structure, morphology and substructure, variation in the parent phase and martensite lattice parameters etc. 
should be considered in a proper way. The effect of some parameters can be followed directly while others act in sophisticated way.

In samples normalized at $T=1270 \mathrm{~K}$ for $\mathrm{t}=20 \mathrm{~min}$. the mean grain size variation during subsequent aging is usually negligible and grain size varies from 20 up to 50 microns. Density of quenched-in vacancies is less than $1 \%$. Dislocation density varies around $10^{-8}-10^{-9} \mathrm{~cm}^{-2}$. Generally speaking in asquenched condition the material structure is relatively homogeneous with locally distributed structural defects. Some of them can annihilate during aging thus improving the structure homogeneity. Since martensite nucleation is favorable around structural defects with an appropriate stress-strain field, this perfect structure homogeneity leads to a decrease of $M_{s}$ temperature and increase of the martesite crystal size. So, the larger the martensite crystals appeared under oriented load, the larger is the transforming vclume shape deformation and recoverable strain. Another source of the recoverable strain variation is the martensite morphology and substructure changes in aged samples. In aged material martensite usually forms in a twinned plate-like form 3-50 nm widths; twinning planes to be presumable $(110)_{\mathrm{M}}$ and $(001)_{\mathrm{M}}$ against $(11 \overline{1})_{M}$ in as-quenched samples $[13,14]$. This is true for the case of alloys with ordinary $\mathrm{B} 2<=>\mathrm{B} 19^{\prime}$ transformation sequence. In alloys with the $\mathrm{B} 2<\Rightarrow \mathrm{R}<\Rightarrow \mathrm{B} 19^{\prime}$ transformation sequence, for nickel content above $\sim 50.5$ at. $\%$, precipitates start to play an important role. Interaction between growing martensite crystals and precipitates (mainly $\mathrm{Ti}_{3} \mathrm{Ni}_{4}$ ) on different stages of their growth leads to wide spectra of variation in the martensite crystal size, morphology and substructure $[3,11]$.

The material strength variation with alloy chemical composition and heat treatment regimes was studied by microhardness $\mathrm{H}_{\mu}$ measurements mainly at room temperature (fig. 6a). Tendency in the binary Ti-Ni alloys strengthening with nickel content (up to $56 \mathrm{at} . \% \mathrm{Ni}$ ) is a well-known phenomena observed by many workers $[3,4,11,15]$. Results differ only in exact hardness value and the critical nickel content. In our measurements a small pseudominimum in the microhardness versus nickel content is observed around 50.3-50.5at.\%Ni. It disappears after a temperature increase up to $\sim 370 \mathrm{~K}$. Since transformation temperatures in these alloys are close to room temperature the microhardness minimum corresponds to martensite formation and stabilization during indentation. Microhardness. of the martensite itself is usually $10-15 \%$ higher than that of the parent phase.

In samples normalized at $\mathrm{T}=1270 \mathrm{~K}$ for $\mathrm{t}=20 \mathrm{~min}$ and quenched in cold water an increase of microhardness is observed for nickel above -50.5 at $\%$, but also depending on the quenching rate. Additional precipitation hardening by $20-25 \%$ takes place after aging at intermediate temperatures (see $2 \mathrm{in}$ fig. 6a). The $\mathrm{Ti}_{3} \mathrm{Ni}_{4}$ particles dominate in alloy hardening at temperatures below $\sim 770 \mathrm{~K}$ due to their higher spatial density as compared with $\mathrm{TiNi}_{3}$ and $\mathrm{Ti}_{2} \mathrm{Ni}_{3}$ particles [16]. (In case of cold-worked samples, an initial stage of recrystallization takes place accompanied by $15-25 \%$ decrease of microhardness [3]).

Increase of microhardness is also observed in binary TiNi alloys doped with some transition metals (fig. 6b). It seems reasonable to adopt that the degree of alloying hardening depends on which base atom sites are occupied by alloying atoms in the B2 lattice. In case of alloying by elements analogous to nickel (for example, $\mathrm{Fe}, \mathrm{Co}$ ) the nickel sites are usually occupied with high probability $\sim 92 \%$ [17]. So, hardenirg increase on substitution of $\mathrm{Fe}$ for $\mathrm{Ni}$ is controlled mainly by cohesive energy while hardening increase on substitution of $\mathrm{Fe}$ for $\mathrm{Ti}$ would be additionally accompanied by precipitation hardening.

\section{CONCLUSIONS}

1. In TiNi-based SMA's, the temperature and stress interval widening of the B2 $<=>\mathrm{R}<=>\mathrm{B} 19^{\prime}$ martensite transformations occurs with increasing nickel content or doping by its chemical analogous $\mathrm{Cr}, \mathrm{Fe}, \mathrm{Co}, \mathrm{Re}$. Reasons of widening are supposed to be increasing material density, decreasing transformation volume dilatation and material hardening due to alloying and precipitates.

2. Response of the TiNi-Me SMA parameters (namely, phase transformation "yield strength", recoverable strain, reversion stress, microhardness, transformation temperatures) on aging condition is usually a nonhomogeneous function of the heat treatment temperature and time. The chosen parameter versus aging temperature or time dependence goes through an extremum specific for a selected property: its position is controlled by the property susceptibility to structure change at different aging stages. 


\section{Acknowledgments}

Part of this work is financially supported by INCO-Copernicus IC15-CT96-0704 (DG 12-MUYS) and INTAS 93-1202 ext. projects. Their contribution is gratefully acknowledged.

\section{References}

[1] Grishkov V.N., Lotkov A.I., "General features of the rhombohedral martensite formation in TiNi alloys", Proceedings of the Conference Martensite-91, Kiev, 1992, 310-313.

[2]Kornilov I.I, Belousov O.K, Kachur E.V. Nitinol and others SMA's. Izd.Nauka, Moscow, 1977,179p

[3]Ermakov V.M., Kolomytsev V.I., Lobodyuk V.A., Khandros L.G. MITOM, 5 (1981) 57-59.

[4]Ermakov V.M., Kolomytsev V.I., Lobodyuk V.A., Musienko R.Ya MITOM, 9 (1989) 61-64.

[5]Kolomytsev V.I. Scripta Metal.Mater., 31, 10 (1994) 1415-1420

[6]Miyazaki S., Otsuka K., Met. Trans. 17A, 1 (1986) 53-63

[7]Miyazaki S., Kimura S., Otsuka K., Phil.Mag.A, 57, 3 (1988) 467-478

[8]Martynov V.V. "Anomalous elasticity, shape memory effect and crystal structure of martensite phases formed by external stresses", Doktorate thesis, Kiev, IMP NASU, 1987, 392 p.

[9]Cherepin A., Lushankin I., Pechkovsky E., Firstov S., Koval Yu. "Fracture of Cu-Al-Ni SMA's" Proceedings of the Conference Martensite-91, Kiev, 1992, 202-205.

[10]Minakov V.N., Tkachuk V.K. "Behaviour of Cu-Al-Ni alloys with martensite structure under various deformation modes", Proceedings of the Conference Martensite-91, Kiev, 1992, 194-197.

[11]Ermakov V.M., Kolomytsev V.I., Lobodyuk V.A., Khandros L.G. Metallofizika, 5 (1982) 23-30

[12]Lin H.C., Wu S-K., Lin J.C. "A study of the martensitic transformation in Ti-rich TiNi alloys", Proc. of the Conference ICOMAT-92, Monterey, California, USA, 1992, 875-880.

[13]Otsuka K. "Crystallography of martensitic transformations and lattice invariant shears". Proc.of the Conference ICOMAT-89, Sydney, Australia, 1990, 393-404.

[14]Matsumoto O., Miyazaki S., Otsuka K., Tamura H., "Crystallographic study of the martensitic transformation in a TiNi alloy" ICOMAT-86, Nara, 1986, 679-684.

[15]Suzuki T., Trans. JIM, 14 (1973) 31.

[16]Kolomytsev V.I., Musienko M.N. "Precipitation processes in TiNi-based SMA's", Proceedings of the XYI Conference Applied Crystallography, 22-24 August 1994,Cieszyn, Poland, 234-241.

[17]Nakata Y., Tadaki T., Shimizu K. Mater.Trans.JIM 327 (1991) 580-586; 32, 12 (1991) 1120-112\% 